\title{
Fluorescence Spectroscopy to Detect Complex Biomolecules in the Plumes of the Outer Solar System: a fast, low-cost, fly-by mission to search for biomarkers.
}

\author{
Corresponding Author: \\ Erin Redwing ${ }^{1}$ \\ eredwing@,berkeley.edu \\ (814)321-5148
}

Co-authors: Christopher P. McKay², Imke de Pater ${ }^{1}$

Co-signees: Francesca Scipioni ${ }^{3}$

${ }^{1}$ University of California, Berkeley, ${ }^{2}$ NASA Ames, ${ }^{3}$ SETI Institute

\begin{abstract}
We propose a conceptually new type of life detection mission for the outer Solar System. Using preliminary radiative transfer calculations, we show that a small spacecraft with a UV laser and fluorescence spectrometer could detect the distinctive signal of aromatic amino acids - particularly tryptophan. Tryptophan is the most complex of the proteinogenic amino acids. The mission we propose would involve a fast trajectory to the Saturn System and a fast flyby of the plume of Enceladus. The mission length and cost would both be much less than current concepts for biomarker detection missions to Enceladus.
\end{abstract}




\section{Introduction}

Enceladus is a target of astrobiological interest due to its active south polar region, where material from its subsurface liquid water ocean erupts via tiger stripe plumes. Analysis of Enceladus' plume by the Cassini spacecraft revealed the presence of salts (Postberg et al. 2011), biologically available nitrogen and carbon (Waite et al. 2009), as well as both simple ( $<50 \mathrm{~g} / \mathrm{mol}$ ) and complex ( $>200 \mathrm{~g} / \mathrm{mol}$ ) organic compounds (Postberg et al. 2018). These findings highlight Enceladus as perhaps the most promising candidate for discovering life on another body in our solar system.

While the discovery of large organic compounds at the plume of Enceladus is exciting, the viability of this broad category as a biosignature varies significantly depending on the nuances of each subcategory of organic compounds. The organic compounds discovered at Enceladus' plume were defined as "complex" due to their high molecular mass, but this alone cannot be used categorically as a proxy for the complexity of the molecule itself (Marshall et al. 2017). Studying a molecule's "complexity" as a biosignature is better understood through Marshall et al. 2017's Pathway Complexity Algorithm, which classifies complexity of biomolecules in terms of the steps required to assemble the molecule from smaller, abiotic building blocks, and finds that molecules created abiotically usually have a pathway complexity value of $<15$ (Marshall et al. 2017). Since the mass spectrometers onboard Cassini could only determine the molar mass and not the actual structure of the molecule, this limits the ability to assess the biosignature potential of the large organic compounds erupting from Enceladus' plume.

One subcategory of organic compounds are amino acids, which are composed of an anime, carboxyl, and side chain group. Over 500 amino acids have been discovered, but only 20 are used in life on Earth. Simple amino acids have been discovered on meteorites and comets (Kvenvolden et al. 1970; Elsila et al. 2009). Of the 20 amino acids used in life on Earth, the most complex is tryptophan (pathway complexity value $=12$ ), an aromatic amino acid essential to protein synthesis in Earth life. Tryptophan (Figure 1) is strongly fluorescent when excited at ultraviolet wavelengths, which allows for it to be easily identified and quantified using fluorescence spectroscopy. Tryptophan fluorescence is widely studied in fields such as biomedicine, where tryptophan has been used as a proxy for Figure 1: Tryptophan changes in protein structure (Vivian and Callis 2001), as well as in ecological studies tracking organic content in seawater samples (Jørgensen et al. 2011). In traditional studies using tryptophan fluorescence, vials of samples are excited with UV light at $266 \mathrm{~nm}$ (peak of tryptophan excitation) and observed in a laboratory setting to quantify visible fluorescence. Tryptophan fluorescence has never been observed remotely, such as what would be required in a spacecraft flyby mission. The primary disadvantage to tryptophan as a target for remote detection is its low natural concentration relative to other aromatic amino acids, such as tyrosine and phenylalanine. In aquatic ecosystems, tyrosine and phenylalanine are 36 and 66 times more abundant, respectively, than tryptophan (Moura et al. 2013). Despite this lower concentration the natural fluorescence of tryptophan is equally detected in proteins compared to tyrosine (Coble 1996, Jameson 2014).

In this white paper, we present preliminary radiative transfer calculations which assess the viability of detecting tryptophan fluorescence via active remote sensing during a high-speed flyby at the plume of Enceladus. We assume reasonable values for the physical and geometrical parameters of a short, close-pass flyby of Enceladus' south polar region, and determine initial estimates of instrumentation requirements for detection of visible fluorescence of tryptophan at a concentration of $1 \mathrm{ppb}$ - a typical concentration in the barren regions of Earth's ocean. Based on 
these results, we propose avenues for future modeling and experimental study of remote tryptophan fluorescence spectroscopy as a biosignature detection method for space-based missions.

There are many advantages to the mission concept proposed here. First, the spacecraft can be small and based on mature (high TRL) technologies - hence low cost. Second, because there is no sampling, nor any need to physically enter the plume, high relative velocities at encounter with the target are permissible. This allows for a fast trajectory with the associated short mission duration. A plausible biomarker detection mission to Enceladus may be achievable on the budget of an Explorer-class mission.

We focus here on Enceladus but there may be other suitable targets in the outer Solar System, such the plumes of Europa or the comae of comets.

\section{Methodology \& Instrumentation}

A generalized setup for a close flyby of the Enceladus south polar region was constructed to illustrate this proposed biosignature detection method - a diagram of which can be seen in Figure 2.

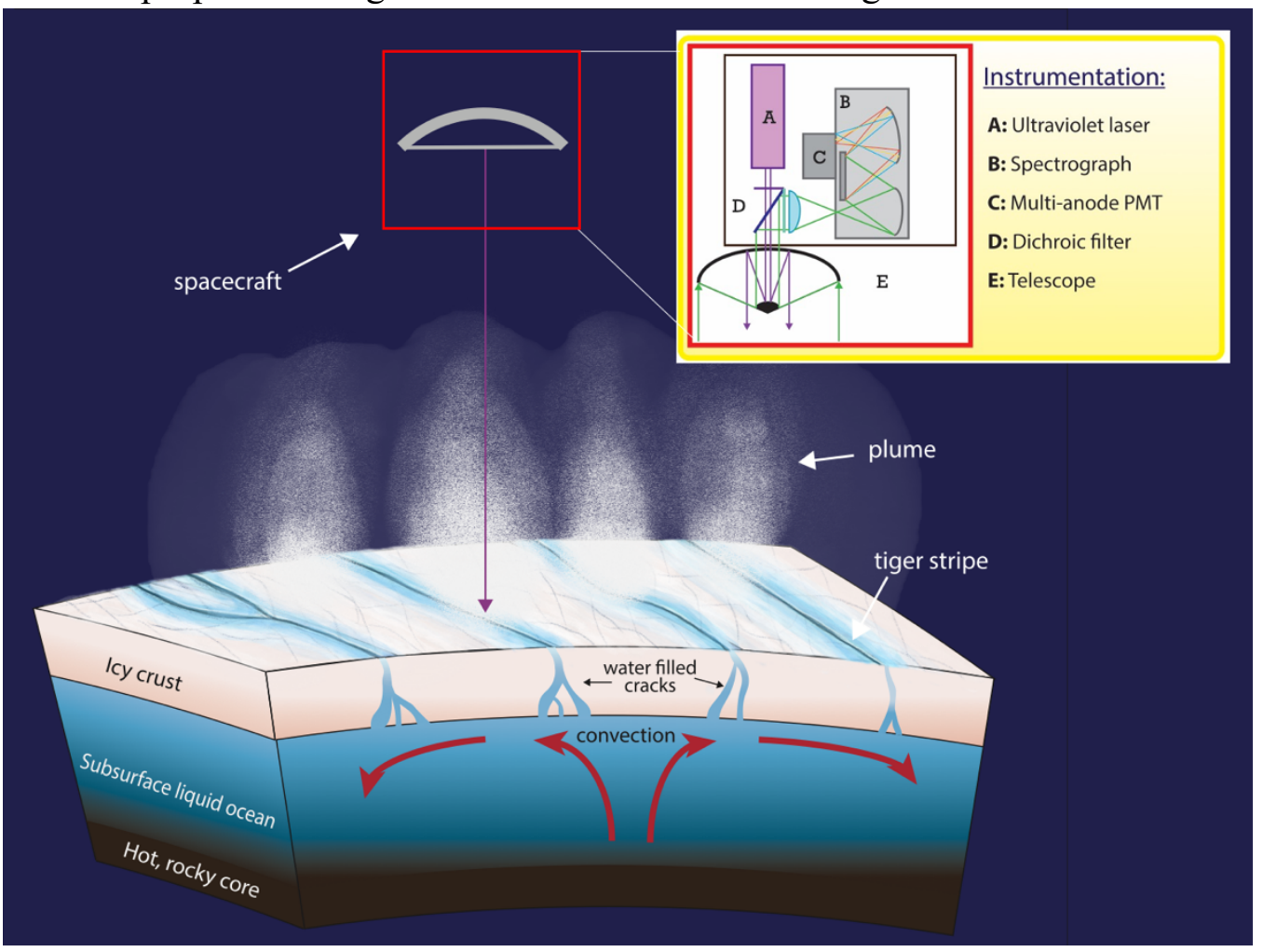

Figure 2: Diagram of Enceladus' internal structure and plume surface features with proposed fly-by configuration and instrumentation.

The instrumentation required is relatively simple: a UV laser at $266 \mathrm{~nm}$ (similar in wavelength as that onboard the SHERLOC instrument on Mars2020) irradiates tryptophan through the telescope optics. The resulting fluorescence is then gathered and focused by the same telescope onto a spectrometer operating in the range of $260-516 \mathrm{~nm}$ which will cover the emission spectrum of the simplest aromatic amino acid (phenylalanine) up through more complex amino acids such as tryptophan, as shown in Figure 3. In this scenario, tryptophan molecules, if present in Enceladus' subsurface ocean and erupted to the surface via its active south polar plumes, would be actively excited by the UV laser, resulting in visible fluorescence which is then recorded by the telescope 
and spectrograph onboard the spacecraft. There are a number of potential laser sources available that could be used and are based on frequency quadrupled fiber or Nd:YAG laser sources. The power requirements of the laser will be highly dependent on the possible flight trajectories. While these sources are not entirely energy-efficient, operation for the short duration through the plume would be possible with suitable battery packs. The spectrometer will be a simple fixed-grating implementation (i.e., no moving parts).

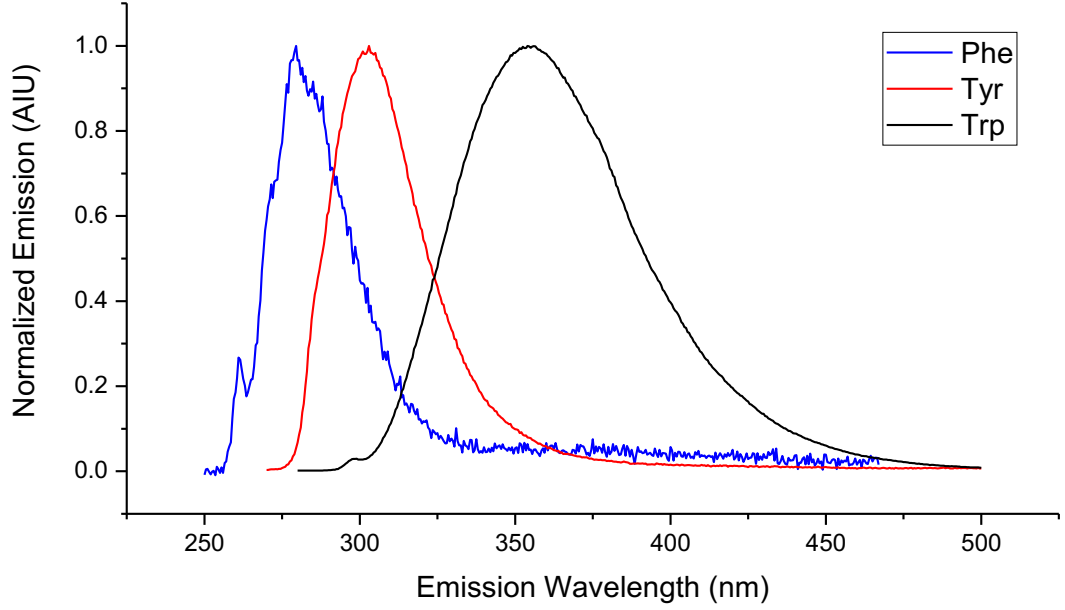

Figure 3: Characteristic emission spectra of phenylalanine (blue), tyrosine (red), and tryptophan (black).
With this theoretical plume flyby set up, the question then becomes a trade-off between the concentration of tryptophan in the material and the laser power required to excite tryptophan fluorescence at a level that can be detected at a reasonable signal to noise ratio. Other than confusion from other fluorescent compounds, the main source of noise will be scattered solar light. This can be minimized by observing the plume at a $90^{\circ}$ phase angle, by the use of polarizers, and pulsing the laser and filtering the detected signal at the same frequency. Ultimately, the noise floor will be set by fluctuations (shot noise) in the solar background.

\section{Derivation Results \& Discussion}

To assess the viability of detecting tryptophan fluorescence given these parameters, two equations of radiative transfer were considered: one for the ultraviolet intensity which is emitted by the laser onboard the spacecraft (Eq.1), and one for the visible intensity which is emitted by tryptophan when it fluoresces (Eq. 2):

$$
\begin{aligned}
& \text { UV: } I(s)=I_{0} e^{-\left(n \sigma+K_{u}\right) s} \\
& \text { Visible: } E(s)=I(s) n \sigma s \varepsilon
\end{aligned}
$$

Where $I(s)$ is the UV intensity as a function of path length $(s)$ through the material, $I_{0}$ is the intensity of the UV laser, $n \sigma$ is the density times the cross-section which thus describes absorption by tryptophan, $K_{u}$ describes scattering of the UV laser through the material (plume ice or surface snow), $E(s)$ is the emission of visible fluorescence of tryptophan, and $\varepsilon$ is tryptophan fluorescence efficiency. UV intensity $(I(s))$ is dependent on the initial laser intensity and drops off as a function of scattering and absorption by tryptophan through the material in question. Visible intensity $(E(s))$ is dependent on the UV light which is absorbed by tryptophan through the material and the efficiency of tryptophan fluorescence. Because the visible fluorescence emission is dependent on the UV intensity, an equation can be set up to solve for the number of visible photons received by the telescope dependent on the power of the UV laser.

In the case where tryptophan is indeed present in Enceladus' subsurface ocean and erupted via the plumes, there would be two potential targets for detecting tryptophan: in the plume itself, and in the snowpack material surrounding the plume where plume materials fall to the surface of 
Enceladus. In terms of radiative transfer, this is essentially the optically-thin scenario (plume) and an optically-thick scenario (bright, reflective snowpack surrounding the plume). Based on preliminary calculations, the plume itself is likely too optically thin to detect tryptophan fluorescence at a reasonable concentration and spacecraft laser power. For this reason, we decided to focus on the snowpack material surrounding the plume for the calculations in this white paper.

The major limitation in the optically-thick, snowpack scenario then becomes scattering of the UV laser through the snowpack itself. Based on UV observations of Enceladus by the Cosmic Origins Spectrograph (COS) on the Hubble Space Telescope (HST), it is assumed that about 85\% of the laser intensity is lost to scattering at $266 \mathrm{~nm}$ (Zastrow et al. 2012). For the purposes of these calculations, we then assume that what is not lost to scattering ( $\sim 15 \%$ of initial laser intensity) is absorbed by tryptophan in the snowpack $\left(I(s) \approx 0.15 I_{0}\right)$. The equation for visible fluorescence of tryptophan (Eq. 2) then becomes:

$$
E(s)=0.15 I_{0} n \sigma s \varepsilon
$$

There is also some geometric dependence: if it is assumed that visible fluoresced photons are emitted in a random spatial distribution then only some of those photons will reach the telescope and be received by the detector. Consider the number of photons which reach the telescope as a ratio of area of the telescope $\left(\pi \mathrm{D}^{2} / 4\right)$, where $\mathrm{D}=$ telescope diameter, to the area of emission $(\pi$ $\left.(\delta+s)^{2}\right)$, where $\delta$ is the flyby distance:

$$
E(s)=0.15 I_{0} n \sigma s \varepsilon * \frac{D^{2}}{4(\delta+s)^{2}}
$$

Rearranging this equation to solve for initial laser intensity $\left(I_{0}\right)$ :

$$
I_{0}=\frac{4 E(s)(\delta+s)^{2}}{0.15 n \sigma s \varepsilon D^{2}}
$$

This is an equation for the number of UV photons emitted by the laser as a function of the number of visible, fluoresced photons received by the telescope. Assuming a close pass flyby $(\delta=10 \mathrm{~km})$ of a spacecraft with a telescope aperture diameter of $1 \mathrm{~m}(\mathrm{D}=1 \mathrm{~m})$, the number density of tryptophan in the snowbank (n) can be calculated assuming the snowbank has a density comparable to freshly fallen snow (Patterson 2016) and that tryptophan is present on an order of magnitude of $1 \mathrm{ppb}$ :

$$
\begin{aligned}
\frac{5 \times 10^{4} \mathrm{~g}}{\mathrm{~m}^{3}} * & \frac{6.02 \times 10^{23} \text { molecules } \mathrm{H}_{2} \mathrm{O}}{18 \mathrm{~g} \mathrm{H}_{2} \mathrm{O}} * \frac{1 \text { molecule tryptophan }}{10^{9} \text { molecules } \mathrm{H}_{2} \mathrm{O}} \\
& =1.67 \times 10^{18} \frac{\text { molecules tryptophan }}{\mathrm{m}^{3}}=n
\end{aligned}
$$

For a reasonable detection, a minimum of 100 visible, fluoresced photons should be received by the spacecraft $(E(s)=100)$. From this, the lower limit on the photons emitted by the spacecraft can be calculated:

$$
I_{0}=\frac{4 E(s)(\delta+s)^{2}}{0.15 n \sigma s \varepsilon D^{2}} \approx 10^{14} \text { photons }
$$

Converting $I_{0}$ to laser energy, and assuming the time length of the flyby is on the order of magnitude of around 10 seconds, the minimum laser power required for detection of tryptophan fluorescence can be determined:

$$
10^{14} \text { photons } * \frac{7.6 \times 10^{-19} \mathrm{~J}}{\text { photon }} * \frac{\text { flyby }}{10 \text { seconds }} \approx 10^{-6}-10^{-5} \mathrm{~W}
$$

Given the parameters outlined in these calculations for a close pass, high speed flyby of Enceladus, the laser power needed to detect tryptophan fluorescence is on the order of $10^{-6}-10^{-5} \mathrm{~W}$. 
While there are many assumptions made in this simple, back of the envelope calculation, the resulting laser power is well within a reasonable limit for a space-based mission and it indicates that this biosignature detection method is worthy of further study. Some of the main assumptions made for these calculations include the following:

1. All absorption of the UV laser in the snowpack is due to tryptophan. In reality, ammonia and water also absorb radiation at this wavelength and would need to be included in more detailed calculations.

2. Tryptophan concentration is uniform throughout the snowpack material (i.e. does not decrease or increase with depth). This would depend on the depth to which plume deposition extends into Enceladus' surface and the degradation of tryptophan molecules in a high-radiation environment.

3. Tryptophan molecules are free and unaffected by other snowbank particles (i.e. tryptophan fluorescence efficiency $=100 \%$ ). The phenomenon of reduced tryptophan fluorescence efficiency (called tryptophan "quenching") is widely studied due to its biomedical applications - changes in the characteristic emission spectrum of tryptophan can be used to indicate the presence of other biomolecules.

\section{Conclusion \& Future Steps}

The calculations described in this paper are simple, but they show the promising potential for this method, a method which is unique and out-of-the-box for traditional space science, but is based on decades-old, tried and true methods from other branches of science. Tryptophan fluorescence spectroscopy is a widely used technique because its fluorescence emission spectra is characteristically distinct (see Figure 3) and strongly recognizable even at extremely small concentrations (Coble 1996). However, the many unknowns surrounding remote tryptophan fluorescence detection, as well as on another planetary body with unknown biological activity, require a great deal of consideration in how the emission spectra may look, and what can be considered to be a reasonable detection. For this reason, it is necessary to model the emission spectra for many scenarios using the constraints and limitations obtained through the calculations presented in this paper. Modeling of tryptophan fluorescence emission spectra for various flyby scenarios would show the viability and limits of detection, which would allow for a better understanding of what is and is not possible in using tryptophan fluorescence as a biosignature detection method.

In addition to modeling, the ubiquity of tryptophan in Earth life allows for the ability to experimentally test out remote detection of tryptophan fluorescence in a variety of scenarios very easily. A simple instrument could be built and tested out at sites on Earth which mimic Enceladus, including icy snowbanks or glaciers. This would give tangible experimental results on the viability of using remote tryptophan fluorescence as a biosignature detection method without needing to build space-based instrumentation. If these experimental trials prove successful, remote detection of tryptophan fluorescence could become a biosignature detection method for future space-based missions and have implications not only for life detection on Enceladus, but other relevant targets such as comets and other icy satellites.

Remote detection of tryptophan fluorescence is a fundamentally different approach to life detection, one that represents an opportunity to reconsider the way we do outer solar system missions. It is a high risk, high reward concept - a small payload on a fast trajectory with no need for orbit insertion burns allows for a mission that is relatively quick and cheap compared to what we have come to expect for missions which target life detection in the outer solar system. The 
search for life in space forces us to think outside the box and consider new approaches to the way we do science, and this proposed biosignature detection method - new to space science but trusted

and ubiquitous in earth-based science - shows the strength in the necessarily interdisciplinary nature of astrobiological studies.

\section{References}

Coble 1996. Marine Chemistry, 51, 325.

Elsila et al. 2009. Meteoritics \& Planetary Science 44, 1323.

Jameson 2014. Introduction to fluorescence. Taylor \& Francis.

Jørgensen et al. 2011. Marine Chemistry, 126, 139.

Kvenvolden et al. 1970. Nature 228, 923.

Marshall et al. 2017. Philosophical Transactions of the Royal Society A: Mathematical, Physical and Engineering Sciences 375.

Moura et al. 2013. PloS one, 8, 77319.

Patterson 2016. The physics of glaciers. Elsevier, 9.

Postberg et al. 2011. Nature 474, 620.

Postberg et al. 2018. Nature 558, 564.

Vivian and Callis 2001. Biophysical Journal 80, 2093.

Waite et al. 2009. Nature 460, 487.

Zastrow et al. 2012. Icarus 220.1: 29-35. 